\title{
Coeficientes de cultura do abacaxizeiro nas condições edafoclimáticas de Uberaba, MG
}

\author{
Márcio J. de Santana ${ }^{1}$, Olegário P. de Souza' ${ }^{1}$, Ayza E.V. Camargos' ${ }^{1}$ J João P.R. Andrade ${ }^{1}$
}

\section{RESUMO}

Dentre as culturas irrigadas o abacaxi vem-se destacando por sua importância econômica e social. É uma cultura exigente em tratos culturais dentre os quais a irrigação exerce forte influência na produção e qualidade dos frutos. O objetivo deste trabalho foi determinar os valores dos coeficientes de cultura e evapotranspiração do abacaxizeiro irrigado e cultivado em Uberaba, MG. O experimento foi conduzido no setor de Fruticultura do IFTM, campus Uberaba, MG. A cultivar utilizada foi a Smooth Cayenne. A irrigação foi realizada por tubogotejadores de duas formas: enterrados e superficiais. Para estimativa da evapotranspiração de referência (ETo) foi utilizada a equação de Penman-Monteith e para obtenção da evapotranspiração da cultura (ETc) o balanço de água no solo. Dentre os resultados verifica-se que o maior valor de evapotranspiração da cultura foi de aproximadamente $6,8 \mathrm{~mm}$; os valores de ETc médios encontrados quando a irrigação foi efetuada com tubogotejadores enterrados e superficiais foram, respectivamente, de 2,88 e 3,37 mm; os valores médios de coeficientes de cultura foram de 0,97 (tubogotejador enterrado) e 0,91 (tubogotejador superficial) para todo o ciclo do abacaxi.

Palavras-chave: Ananas comosus (L.) Merril, manejo de irrigação, Kc

\section{Pineapple crop coefficient under edaphoclimatic conditions of Uberaba, MG}

\begin{abstract}
Among the irrigated crops, the pineapple stand out for both its economic and social importance. It is a crop demanding in cultural practices, among which irrigation exercises strong influence upon the production and quality of fruits. The purpose of this study was to determine the values of the crop coefficients and evapotranspiration of the pineapple crop in Uberaba, MG. The experiment was conducted in the Fruit Crops Sector of "Instituto Federal de Educação, Ciência e Tecnologia do Triângulo Mineiro, campus Uberaba", MG. The cultivar used was Smooth Cayenne. Irrigation was carried out through driplines in two manners: buried and surface. For the estimate of the reference evapotranspiration (ETo), the Penman-Monteith equation was utilized and for obtaining the crop evapotranspiration (ETC), the soil water balance was used. Among the results, the highest value of evapotranspiration of the crop was about $6.8 \mathrm{~mm}$; the values of mean ETc found when irrigation was performed with buried and surface driplines were respectively of 2.88 and $3.37 \mathrm{~mm}$ and when the irrigation was performed with surface and buried piping, respectively; the mean values of crop coefficient were of 0.97 (driplines buried) and 0.91 (driplines surface) for the entire cycle of the pineapple crop.
\end{abstract}

Key words: Ananas comosus (L.) Merril, irrigation management, Kc 


\section{INTRODUÇÃO}

O abacaxizeiro (Ananas comosus (L.) Merril) pertence à família Bromeliaceae é originário da América do Sul, provavelmente das regiões Sul e Sudeste do Brasil, Argentina e Uruguai. Tailândia, Brasil e Filipinas se destacam como os principais países produtores (Ponciano et al., 2006) seguidos dos Estados Unidos, China e Índia (Melo et al., 2006). Quanto aos tratos culturais a irrigação é essencial para aumentar a produção, ter melhor padronização dos frutos e melhorar sua qualidade resultando em maior retorno econômico, além de permitir colocar frutos no mercado no período de entressafra e exploração de uma segunda safra, aumentando em até $30 \%$ a produtividade comparada ao cultivo de sequeiro (Souza et al., 2007). E tem como consequência positiva, o aumento da produtividade e da rentabilidade (Bengozi et aL., 2007).

Com a expansão do abacaxizeiro irrigado no país, tanto em regiões tradicionalmente produtoras quanto em novas áreas de plantio, aumentaram as demandas por tecnologias e informações que permitem o manejo adequado da cultura. A maioria das áreas produtoras de abacaxi está sujeita a períodos secos, o que ocasiona déficit hídrico para a cultura. Nessas regiões o uso da irrigação e de densidades adequadas, é importante para a elevação da produtividade e para a melhoria da qualidade dos frutos. Mesmo sendo considerada uma cultura relativamente adaptada ao déficit hídrico, a irrigação é fundamental para uma produção mais uniforme. Nas regiões tropicais brasileiras a irrigação tem sido usada de forma complementar atendendo à demanda hídrica das plantas nos meses com menores índices de chuva, compreendendo o período de maio a setembro (Silva \& Silva, 2006).

Dentre os métodos empregados na irrigação, a irrigação por gotejamento superficial é o método que mais se desenvolveu e expandiu nos últimos anos em razão da sua alta eficiência no uso da água. A utilização do gotejamento subterrâneo adicionado às vantagens do gotejamento superficial, evita que a demanda evaporativa da atmosfera interfira na distribuição de íons no bulbo molhado reduzindo o acúmulo de sais fertilizantes na superfície do solo em torno do ponto de emissão propiciando, desta forma, uma produtividade melhor das culturas em função da melhor distribuição espacial desses íons no sistema radicular da planta (Monteiro et al., 2007).

Nas áreas de maior concentração de produção de abacaxi no Triângulo Mineiro, a estação chuvosa se concentra de outubro a março; entretanto, ocorre período seco de abril a setembro, o que leva as lavouras de sequeiro a apresentar baixo rendimento e frutos de qualidade inferior. As fases críticas para a cultura se concentram no período de crescimento vegetativo, da floração à colheita, visto que o déficit hídrico pode afetar a produção, o peso do fruto e a qualidade (Carvalho et al., 2005).

Existem poucas informações disponíveis sobre as relações entre o consumo de água, o crescimento e a produtividade na cultura do abacaxi (Melo et al., 2006). Outros aspectos, como os coeficientes de cultura, também necessitam de informações para esta importante região produtora. O coeficiente de cultura
(Kc) vem sendo empregado com o objetivo de obtenção da evapotranspiração das culturas pois nem sempre são encontrados valores para determinados locais ou cultivares. Apesar da importância econômica da cultura do abacaxi para as regiões com potencial edafoclimático apropriado para seu cultivo, pesquisas relacionadas aos seus aspectos agrometeorológicos ainda são incipientes (Souza et al., 2008). Ante o exposto o objetivo do trabalho foi determinar os valores dos coeficientes de cultura $(\mathrm{Kc})$ e a evapotranspiração do abacaxizeiro cultivado em Uberaba, MG.

\section{Material e MÉtodos}

O trabalho foi conduzido no setor de Fruticultura do Instituto Federal de Educação, Ciência e Tecnologia do Triângulo Mineiro, Campus Uberaba, localizado no município de Uberaba, MG, situado a $19^{\circ} 39^{\prime} 19^{\prime \prime} \mathrm{S}$ e $47^{\circ} 57^{\prime} 27^{\prime \prime} \mathrm{W}$. O solo é de topografia plana, do tipo Latossolo Vermelho distroférrico e textura franco-arenosa.

A cultivar utilizada foi o Smooth Cayenne, cujas mudas do tipo rebentão pesando aproximadamente $400 \mathrm{~g}$. As mudas passaram por uma "cura" durante duas semanas, antes do plantio. Um tratamento foi realizado com fungicida (Thiophanate methyl) e inseticida (Tiametoxam) como medida preventiva ao fungo Fusarium subglutinans (Fusariose) e para a cochonilha (Dysmicocus brevipes).

Quanto ao preparo do solo foi realizada uma aração a 0,3 $\mathrm{m}$ de profundidade seguida de uma gradagem. As adubações de plantio e de cobertura foram feitas de acordo com a análise química do solo, seguindo as recomendações de Souza et al. (2012). Durante a condução do experimento foram efetuados os tratos culturais e fitossanitários preconizados para a cultura. Visando à uniformização da época de produção, a indução floral foi realizada com solução de Ethrel mais ureia $2 \%$, aplicada na roseta foliar.

As equações de ajuste das curvas características de retenção da água no solo para as camadas de 0-20 e 20-40 $\mathrm{cm}$ de profundidade foram obtidas cujos parâmetros estão encontram na Tabela 1. Com auxílio do software SWRC versão 3.0 (Soil Water Retention Curve) obtiveram-se os parâmetros de ajuste das equações. A obtenção da curva característica de água no solo foi realizada em amostras deformadas no Laboratório de Relação Solo-Planta, do IFTM, Campus Uberaba, MG. Para as tensões de 2, 4, 6, 8 e $10 \mathrm{kPa}$ foi utilizado o método do funil de placa porosa (funil de Hainess) e para as tensões de 33, 100, 500 e 1500 $\mathrm{kPa}$, a câmara de pressão de Richards.

A densidade média do solo para as camadas de 0-20 e 20$40 \mathrm{~cm}$, obtida pelo método do cilindro de Uhland, forneceu valores de 1,15 e $1,2 \mathrm{~kg} \mathrm{dm}^{-3}$, respectivamente. A umidade

Tabela 1. Caracterização hídrica do solo da área experimental

\begin{tabular}{ccccc}
\hline Camada & \multicolumn{4}{c}{ Parâmetros } \\
\cline { 2 - 5 } (cm) & $\mathbf{\theta r}$ & $\mathbf{\theta s}$ & $\mathbf{~ m}$ & $\mathbf{n}$ \\
$0-20$ & 0,115 & 0,305 & 0,11 & 4,60 \\
$20-40$ & 0,225 & 0,115 & 0,13 & 6,83 \\
\hline
\end{tabular}

$\theta$ r e $\theta \mathrm{r}$ - umidade residual e de saturação $\left(\mathrm{cm}^{3} \mathrm{~cm}^{-3}\right)$; $\mathrm{m}$ e $\mathrm{n}$ parâmetros independentes de ajuste equação de Genuchten (adimensional) 
correspondente à capacidade de campo foi de $0,23 \mathrm{~cm}^{3} \mathrm{~cm}^{-3}$ (tensão de água no solo média de $10 \mathrm{kPa}$ na camada de $0-20 \mathrm{~cm}$ ).

A irrigação foi realizada de duas formas: com tubogotejadores enterrados (a $0,10 \mathrm{~m}$ de profundidade) e superficiais. $\mathrm{O}$ manejo da irrigação foi efetuado em turno de rega de 2 dias, por meio de tensiometria (tensímetro de punção). Os dados de balanço de água no solo foram coletados em seis parcelas. A parcela experimental foi constituída de fileiras duplas, com 3,6 m de comprimento, 1,40 m de largura e densidade de 3,3 plantas por metro. Na linha, as plantas ficaram dispostas em quincôncio em espaçamento de $90 \times 50 \times 30 \mathrm{~cm}$. O plantio foi realizado em novembro de 2010 e a colheita em dezembro de 2012.

Baterias de tensiômetros foram instaladas a 0,10 e $0,30 \mathrm{~m}$ de profundidade em 6 (seis) parcelas experimentais (três em cada forma de irrigação). Com as tensões observadas foram calculadas as umidades correspondentes, a partir da curva característica (Tabela 1). De posse dessas umidades e com a correspondente à capacidade de campo e se considerando ainda a profundidade do sistema radicular de $0,3 \mathrm{~m}$, calcularam-se as lâminas de irrigação (Eqs. 1, 2 e 3).

$$
\begin{gathered}
\mathrm{LL}=\left(\theta_{\mathrm{cc}}-\theta_{\text {atual }}\right) \times \mathrm{z} \\
\mathrm{LB}=\frac{\mathrm{LL}}{(1-\mathrm{k}) \times \mathrm{CU}} \\
\mathrm{MB}_{\text {media }}=\mathrm{MB}_{\text {media } 10 \mathrm{~cm}}+\mathrm{LB}_{\text {media } 30 \mathrm{~cm}} \\
\mathrm{~K}=1-\mathrm{Ea} \\
\mathrm{k}=\mathrm{LR}=\frac{\mathrm{CEa}}{(2 \times \mathrm{CEe})} \\
\mathrm{T}=\frac{\mathrm{LB}{ }_{\text {media }} \times \mathrm{A}}{\mathrm{e} \times \mathrm{qa}}
\end{gathered}
$$

em que:

LL - lâmina líquida de irrigação em cada subcamada, $\mathrm{mm}$

$\theta_{c c} \quad$ - umidade na capacidade de campo, $\mathrm{cm}^{3} \mathrm{~cm}^{-3}$

$\theta_{\text {atual }}^{\text {cc }}$ - umidade no momento de irrigar, $\mathrm{cm}^{3} \mathrm{~cm}^{-3}$

$\mathrm{z}$ - profundidade do sistema radicular, $\mathrm{mm}$

LB - lâmina bruta de irrigação, mm

$\mathrm{k}$ - constante que leva em consideração a salinização do solo e a eficiência de aplicação do sistema

Ea - eficiência de aplicação de água do sistema de irrigação $(0,90)$

LR - lâmina necessária para lavagem do solo, $\mathrm{mm}$

$\mathrm{CEa}$ - condutividade elétrica da água de irrigação, $\mathrm{dS} \mathrm{m}^{-1}$

CEe - condutividade elétrica do extrato de saturação do solo, $\mathrm{dS} \mathrm{m}{ }^{-1}$

CU - coeficiente de uniformidade $(0,92)$

$\mathrm{LB}_{\text {media } 10 \mathrm{~cm}}$ - lâmina obtida pela média das leituras dos sensores instalados a $0,10 \mathrm{~m}$

$\mathrm{LB}_{\text {media } 30 \mathrm{~cm}}$ - lâmina obtida pela média das leituras dos sensores instalados a $0,30 \mathrm{~m}$

$\mathrm{T}$ - tempo de funcionamento do sistema de irrigação em cada tratamento

A - área ocupada por planta, $\mathrm{m}^{2}$ e - número de emissores por planta

qa - vazão média dos emissores, $\mathrm{L} \mathrm{h}^{-1}$

Próximo à área experimental foi instalada uma estação meteorológica automática, com sensores de velocidade do vento, precipitação pluvial, umidade relativa do ar, temperatura e radiação solar; a coleta diária dos dados foi realizada às $9 \mathrm{~h}$.

Estimou-se a evapotranspiração de referência aplicando-se a equação de Penman-Monteith (Eq. 7) Allen et al. (1998).

$$
\text { ETo }=\frac{0,408 \Delta(\mathrm{Rn}-\mathrm{G})+\gamma \frac{900}{(\mathrm{~T}+273)} \mathrm{U}_{2}(\mathrm{es}-\mathrm{ea})}{\Delta+\gamma\left(1+0,34 \mathrm{U}_{2}\right)}
$$

em que:

ETo - evapotranspiração de referência, $\mathrm{mm} \mathrm{d}^{-1}$

$\Delta$ - declividade da curva de pressão de vapor na saturação, $\mathrm{kPa}^{\circ} \mathrm{C}^{-1}$

$\mathrm{Rn}$ - saldo de radiação, $\mathrm{MJ} \mathrm{m}^{2} \mathrm{~d}^{-1}$

$\gamma \quad$ - constante psicrométrica, $\mathrm{kPa}^{\circ} \mathrm{C}^{-1}$

$\mathrm{G} \quad$ - fluxo de calor no solo, $\mathrm{kPa}^{\circ} \mathrm{C}^{-1}$

$\mathrm{U}_{2}$ - velocidade do vento a $2 \mathrm{~m}$ de altura, $\mathrm{m} \mathrm{s}^{-1}$

$\mathrm{T}$ - temperatura média, ${ }^{\circ} \mathrm{C}$

es - pressão de vapor na saturação, $\mathrm{kPa}$

ea - pressão de vapor atual, $\mathrm{kPa}$

A evapotranspiração da cultura foi estimada por meio do balanço de água no solo (Reichardt, 1985) em um volume de controle correspondente à profundidade de $0,4 \mathrm{~m}$ (Eq. 8).

$$
\Delta \mathrm{h}=\mathrm{P}+\mathrm{I} \pm \mathrm{Q}-\mathrm{ETc}-\mathrm{E}
$$

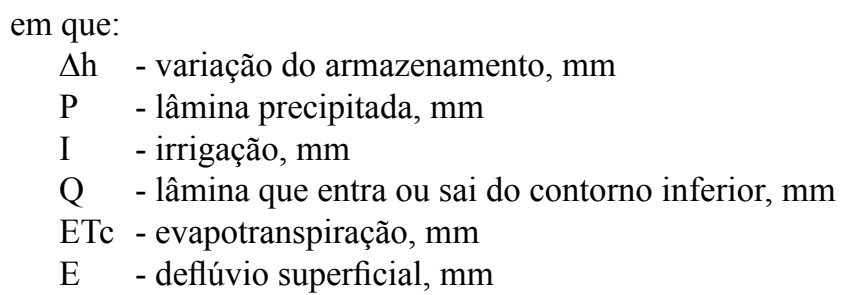

Para o cálculo do deflúvio superficial (E) foram confrontadas as lâminas precipitadas com a lâmina infiltrada potencial, fornecida pela equação de infiltração acumulada do solo, a qual foi estimada com dados obtidos pelo método do infiltrômetro de anel, com base no modelo do tipo potencial (Eq. 9):

$$
\mathrm{I}=\mathrm{aT}^{\mathrm{n}}
$$

em que:

I - infiltração acumulada, L

a - parâmetro do solo, dependente da condição inicial de umidade, $\mathrm{L} \mathrm{T}^{-\mathrm{n}}$

T - tempo de infiltração, T

n - parâmetro característico do solo, adimensional e constante

O experimento foi instalado em uma área considerada plana resultando em um escoamento superficial praticamente nulo. 
O movimento de água no contorno inferior (Q) foi determinado pela Equação de Darcy-Buckingham (Eq. 10):

$$
q=-K(\theta) \frac{d \psi}{d x}
$$

em que:

$\mathrm{q} \quad$ - densidade de fluxo da água no solo, $\mathrm{mm} \mathrm{h}^{-1}$

$\mathrm{K}(\theta)$ - condutividade hidráulica do solo, $\mathrm{mm} \mathrm{h}^{-1}$

$\mathrm{d} \psi / \mathrm{dx}$ - gradiente de potencial total, $\mathrm{mm} \mathrm{mm}^{-1}$

A condutividade hidráulica do solo não saturado foi determinada conforme a Eq. 11 .

$$
\mathrm{K}(\theta)=\mathrm{K}_{0} \mathrm{~W}^{\mathrm{L}}\left[1-\left(1-\mathrm{w}^{\frac{1}{\mathrm{~m}}}\right)^{\mathrm{m}}\right]^{2}
$$

em que:

w - saturação relativa sendo igual a $(\theta-\theta \mathrm{r}) /(\theta \mathrm{s}-\theta \mathrm{r})$

$\mathrm{K}_{\mathrm{o}} \quad$ - condutividade hidráulica do solo saturado

L - parâmetro empírico, sendo de 0,5 para a maioria dos solos

$\theta \quad$ - umidade atual do solo, $\mathrm{cm}^{3} \mathrm{~cm}^{-3}$

$\theta \mathrm{r} \quad$ - umidade residual do solo, $\mathrm{cm}^{3} \mathrm{~cm}^{-3}$

$\theta \mathrm{s} \quad$ - umidade de saturação do solo, $\mathrm{cm}^{3} \mathrm{~cm}^{-3}$

Na obtenção da condutividade hidráulica do solo saturado $\left(\mathrm{K}_{0}\right)$ foi utilizado o Permeâmetro tipo Guelph (no local experimental realizando-se três repetições). A variação do armazenamento foi calculada com base na Eq. 12 considerandose a profundidade igual a $0,4 \mathrm{~m}$.

$$
\Delta \mathrm{h}=\left(\theta_{2}-\theta_{1}\right) \mathrm{z}
$$

em que:

$\Delta \mathrm{h}$ - variação de armazenamento no intervalo de tempo considerado, mm

$\theta_{2} \quad$ - umidade média no tempo final, $\mathrm{cm}^{3} \mathrm{~cm}^{-3}$

$\theta_{1} \quad$ - umidade média no tempo inicial, $\mathrm{cm}^{3} \mathrm{~cm}^{-3}$

z $\quad$ profundidade considerada para o balanço, $400 \mathrm{~mm}$

O coeficiente de cultura Kc foi determinado conforme Eq. 13.

$$
\mathrm{Kc}=\frac{\mathrm{ETc}}{\mathrm{ETo}}
$$

em que:

ETc - evapotranspiração da cultura, $\mathrm{mm} \mathrm{d}^{-1}$

ETo - evapotranspiração de referência, $\mathrm{mm} \mathrm{d}^{-1}$.

\section{Resultados E Discussão}

Na Figura 1 se encontram os valores médios de tensões da água no solo $(\mathrm{kPa})$. Maiores valores de tensão da água no solo foram verificados a partir dos 160 dias após o plantio (DAP) tanto nas parcelas irrigadas com tubulação superficial quanto em tubulação enterrada. Em maiores profundidades $(0,30 \mathrm{~m})$ as tensões verificadas foram, em média, superiores às tensões registradas a $0,1 \mathrm{~m}$.

\section{A. Tubulação superficial}

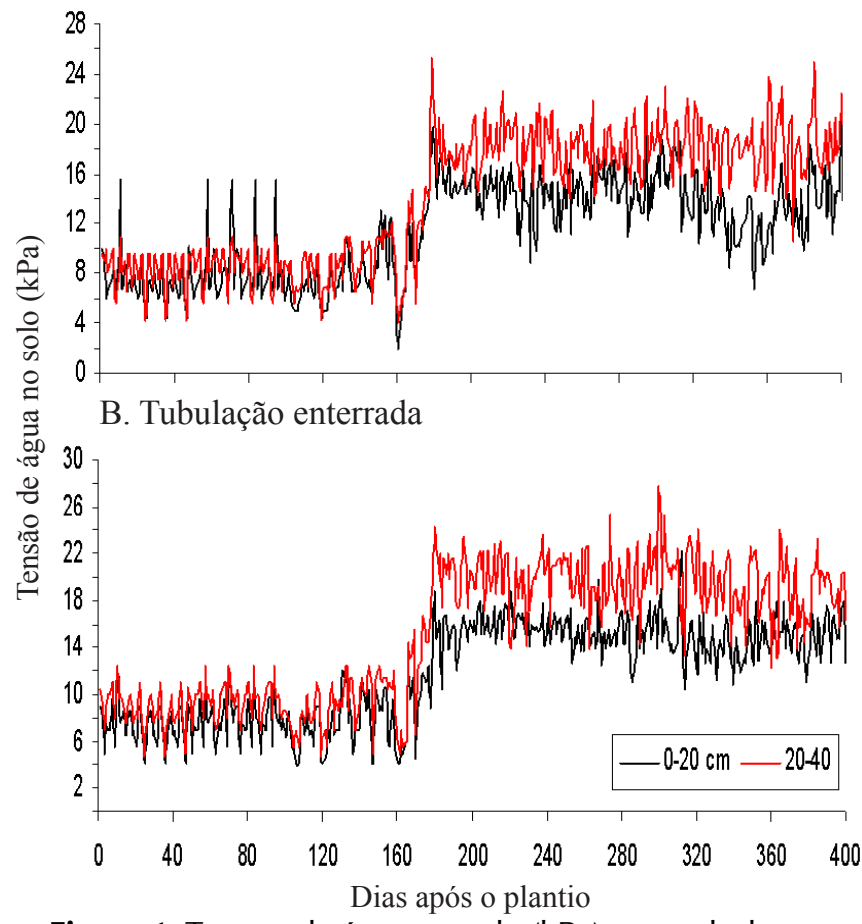

Figura 1. Tensão de água no solo $(\mathrm{kPa})$ para tubulação superficial (A) e enterrada (B)

Observaram-se, pela Figura 2A, os valores de evapotranspiração da cultura quando a irrigação foi efetuada com tubogotejadores enterrados e superficiais. Menores valores de ETc foram verificados próximos aos 210 dias após plantio (DAP) em função desta época ser a mais fria em Uberaba, MG (meses de junho e julho). O valor médio de ETc para tubulação enterrada foi $2,88 \mathrm{~mm}$ e superficial de 3,37 mm. A evapotranspiração de referência foi maior até aproximadamente os 120 dias; a partir desta data houve uma redução ocasionada, provavelmente, pela queda da temperatura (Figura 2B). A evapotranspiração de referência média verificada foi de 4,9 mm. As necessidades hídricas do abacaxizeiro estão ligadas às condições climáticas, umidade do solo e estádio de desenvolvimento da planta. A demanda diária de água pode variar de 1,3 a 5,0 mm (Rotondano \& Melo, 2011).

Os valores diários para ETc estão na Figura 3. Os valores médios de ETc quando a irrigação foi efetuada com tubulação superficial e enterrada em cada estádio da culturas, são apresentados na Tabela 2. Notaram-se maiores valores quando os tubogotejadores foram colocados superficialmente no solo. É provável que a evaporação superficial da água tenha favorecido esta diferença. A ETc foi maior na fase inicial em virtude da cultura ter sido implantada na primavera/verão épocas essas com maior radiação e perda de água no solo. Nota-se que no último estádio o valor de ETc volta a aumentar devido não apenas à época climática mas também à fase de produção da 


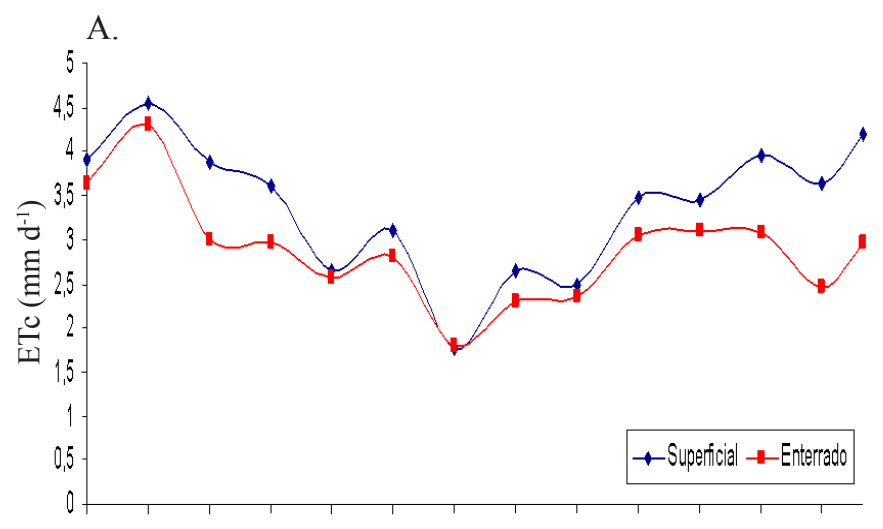
B.

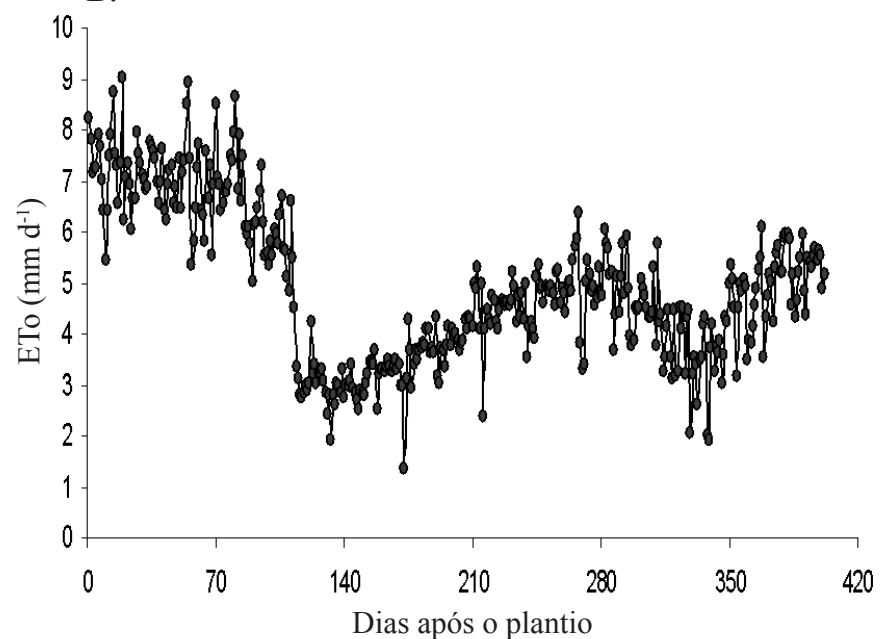

Figura 2. Evapotranspiração da cultura - médias decendiais (A) e evapotranspiração de referência (B)

Tabela 2. Evapotranspiração média da cultura para diferentes estádios de desenvolvimento

\begin{tabular}{|c|c|c|c|}
\hline \multirow{2}{*}{ Estádios } & \multirow{2}{*}{$\begin{array}{c}\text { Duração } \\
\text { (DAP) }\end{array}$} & \multicolumn{2}{|c|}{ ETc tubulação $\left(\mathrm{mm} \mathrm{d}^{-1}\right)$} \\
\hline & & Superficial & Enterrada \\
\hline Inicial & 1 a 60 & 4,22 & 3,98 \\
\hline Desenvolvimento vegetativo & 61 a 210 & 2,99 & 2,62 \\
\hline Intermediário ou de produção & 211 a 300 & 2,86 & 2,56 \\
\hline Final ou de maturação & 301 a 402 & 3,73 & 2,87 \\
\hline
\end{tabular}

cultura.Segundo Souza et al. (2009) a evapotranspiração média total do abacaxizeiro quando utilizado o método do tanque classe A, foi de $2,45 \mathrm{~mm}$.

$\mathrm{Na}$ Tabela 3 estão os valores médios estimados dos coeficientes de cultura para o abacaxizeiro. Quando comparados com os valores obtidos por Almeida (1995) pode-se observar que apenas no estádio inicial e no desenvolvimento vegetativo foram semelhantes e que houve diferenciação para o estádio

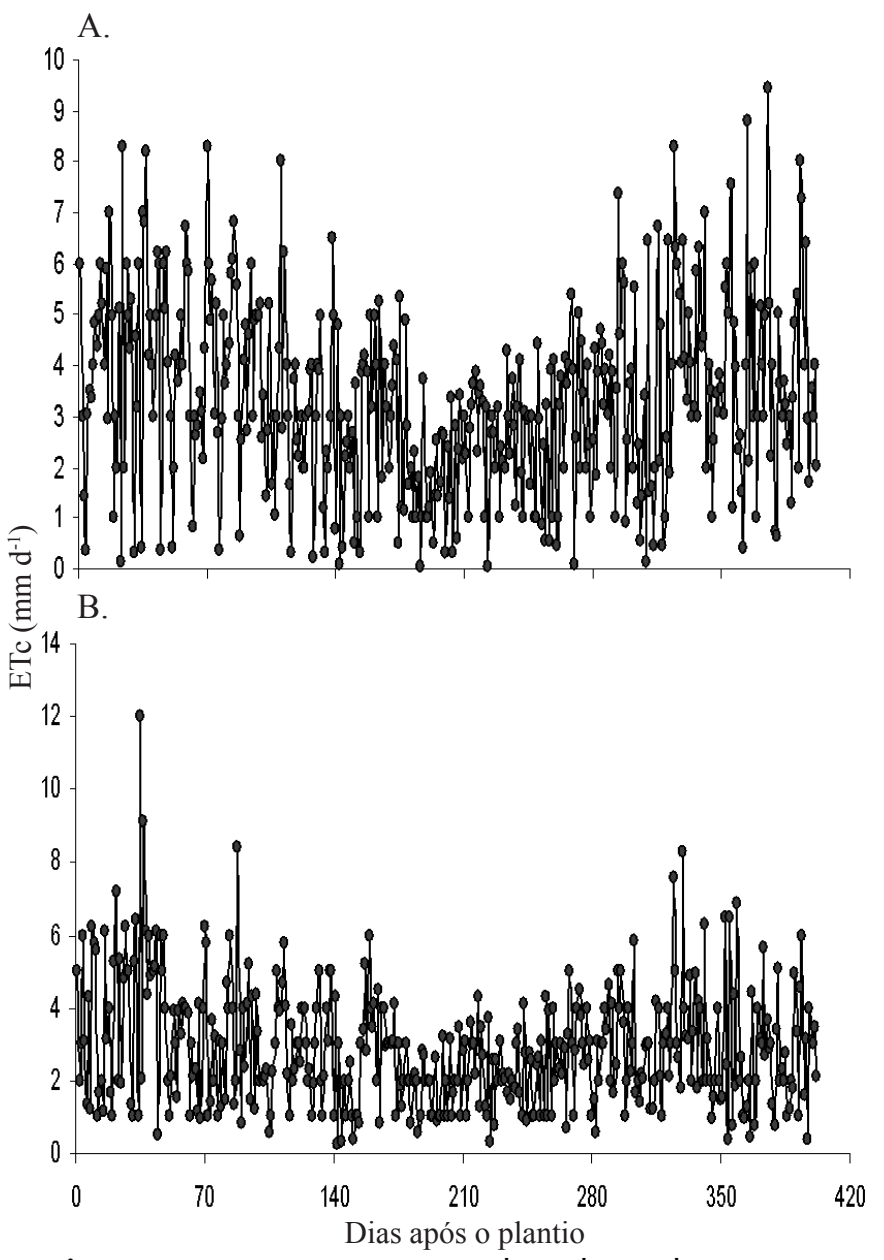

Figura 3. Evapotranspiração da cultura diária para tubulação superficial (A) e enterrada (B)

intermediário ou de produção, tanto quanto para o estádio final ou de maturação. Ressalta-se que as condições de solo e clima condicionam valores de $\mathrm{Kc}$ para as diferentes fases, cultivares $\mathrm{e}$ regiões. Na Tabela 4 se acham os valores médios dos coeficientes de cultura para cada mês de duração do ciclo da cultura; nota-se que no décimo segundo mês (outubro), maior valor de $\operatorname{Kc}(1,13)$ se deve à fase de enchimento do fruto e maturação. Souza et al. (2012) utilizaram valores de Kc de 0,4 para a fase inicial (até os 60 dias após plantio) com vista à condução da cultura irrigada em campo. Esses autores acresceram 0,004 diariamente no valor inicial de Kc no período de desenvolvimento vegetativo (61 aos 210 dias); posteriormente, entre os 211 e 300 dias após o plantio acresceram 0,002222 diariamente nos valores de Kc. Em pesquisa realizada na Paraíba com a cultivar Pérola, Azevedo et al. (2007) determinaram valores de evapotranspiração e coeficientes de cultura; no crescimento vegetativo os autores encontraram um valor de ETc de 4,6 $\mathrm{mm} \mathrm{d}^{-1}$ e de $3,5 \mathrm{~mm} \mathrm{~d}^{-1}$

Tabela 3. Coeficientes de cultura médios para diferentes estádios de desenvolvimento do abacaxizeiro

\begin{tabular}{|c|c|c|c|c|}
\hline \multirow{2}{*}{ Estádios } & \multirow{2}{*}{$\begin{array}{c}\text { Duração } \\
\text { (DAP) }\end{array}$} & \multirow{2}{*}{$\begin{array}{c}\text { Almeida } \\
\text { (1995) }\end{array}$} & \multicolumn{2}{|c|}{ Atual experimento - Kc tubulação } \\
\hline & & & Superficial & Enterrada \\
\hline Inicial & 1 a 60 & $0,4-0,6$ & 0,60 & 0,57 \\
\hline Desenvolvimento vegetativo & 61 a 210 & Varia linearmente entre os valores do $1^{\circ}$ ao $3^{\circ}$ estádios & 0,73 & 0,66 \\
\hline Intermediário ou de produção & 211 a 300 & $1,0-1,2$ & 0,62 & 0,56 \\
\hline Final ou de maturação & 301 a 402 & Varia linearmente entre $03^{\circ}$ estádio e 0,4 a 0,6 & 0,90 & 0,71 \\
\hline
\end{tabular}


Tabela 4. Coeficientes de cultura médios do abacaxizeiro de acordo com o ciclo da cultura (em cada mês)

\begin{tabular}{cccc}
\hline \multirow{2}{*}{ Mês } & \multirow{2}{*}{ DAP } & \multicolumn{2}{c}{ Kc tubulação } \\
\cline { 3 - 4 } 1 & 30 & Superficial & Enterrada \\
2 & 60 & 0,55 & 0,52 \\
3 & 90 & 0,63 & 0,61 \\
4 & 120 & 0,59 & 0,46 \\
5 & 150 & 0,75 & 0,65 \\
6 & 180 & 0,91 & 0,85 \\
7 & 210 & 0,99 & 0,94 \\
8 & 240 & 0,50 & 0,50 \\
9 & 270 & 0,63 & 0,54 \\
10 & 300 & 0,57 & 0,56 \\
11 & 330 & 0,71 & 0,62 \\
12 & 360 & 0,73 & 0,64 \\
13 & 390 & 1,13 & 0,88 \\
14 & 402 & 0,75 & 0,51 \\
\hline
\end{tabular}

DAP - dias após o plantio

na fase de colheita dos frutos. Ainda para os mesmos autores, os menores valores de $\mathrm{Kc}$ foram observados na fase de desenvolvimento vegetativo (aproximadamente 0,88 ).

Na Figura 4 são apresentados coeficientes de cultura para o abacaxizeiro irrigado com tubulação superficial e tubulação enterrada sendo que os Kcs para o abacaxizeiro sob tubulação enterrada apresentaram menores valores em razão, possivelmente, da menor perda de água ocorrida. $\mathrm{Na}$ tubulação superficial foram verificados valores médios de Kc entre 0,5 a 1,1, e para a tubulação enterrada valores médios de Kc compreendidos entre 0,4 a 0,95 .

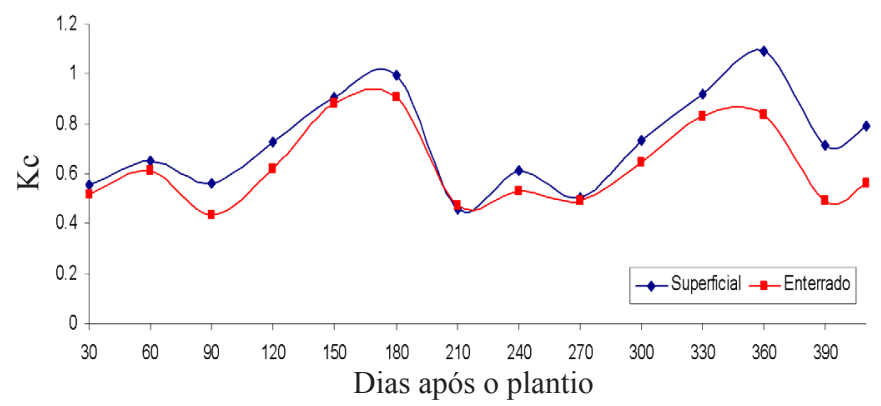

Figura 4. Coeficientes de cultura encontrados em irrigação com tubulação superficial e enterrada

\section{Conclusões}

1. Os maiores valores de evapotranspiração encontrados na cultura do abacaxizeiro foram para os três primeiros meses de plantio devido à época de plantio e na época de maturação da cultura; referidos valores médios diários foram de 4,22;2,99;2,86 e 3,73 mm (tubulação superficial) e de 3,98; 2,62; 2,56 e 2,87 mm (tubulação enterrada) nos estádios de desenvolvimento inicial, desenvolvimento vegetativo, produção e maturação, respectivamente.

2. Os maiores valores de coeficiente de cultura foram verificados na fase de maturação sendo de 0,90 e 0,71 (tubo gotejadores superficiais e tubo gotejadores enterrados, respectivamente).

\section{Agradecimentos}

À FAPEMIG, pelo apoio financeiro ao projeto e à bolsa de pesquisa; enfim, ao CNPq, pela bolsa de iniciação científica cedida.

\section{Literatura Citada}

Allen, R. G.; Pereira, L. P.; Raes, D.; Smith, M. Crop evapotranspiration: guidelines for computing crop water requirements. Rome: FAO, 1998. 300p. FAO, 56

Almeida, O. A. Irrigação. In: Reinhardt, D. H.; Souza, L. F. da S.; Cabral, J. R. S. (org.) Abacaxi: Produção aspectos técnicos. Cruz das Almas: Embrapa Mandioca e Fruticultura 1995. p.35-40.

Azevedo, P. V. de; Souza, C. B.; Silva, B. B. da; Silva, V. de P. R. da. Water requirements of pineapple crop grown in a tropical environment, Brazil. Agricultural Water Management, v.88, p.201-208, 2007.

Bengozi, F. J.; Sampaio, A. C.; Gutierrez, A. D. De S.; Rodrigues, V. M.; Pallamin, M. L. Qualidades físicas e químicas do abacaxi comercializado na CEAGESP, São Paulo. Revista Brasileira de Fruticultura, v.29, p.540-545, 2007.

Carvalho, S.L.C. de; Neves, C. S. V. J.; Bürkle, R.; Marur, C. J. Épocas de indução floral e soma térmica do período do florescimento à colheita de abacaxi 'Smooth Cayenne'. Revista Brasileira de Fruticultura, v.27, p.430-433, 2005.

Melo, A.S.; Aguiar Neto, A.O.; Dantas Neto, J.; Brito, M. E. B.; Viégas, P. R. A.; Magalhães, L. T. S.; Fernandes, P. D. Desenvolvimento vegetativo, rendimento da fruta e otimização do abacaxizeiro cv. Pérola em diferentes níveis de irrigação. Ciência Rural, v.36, p.93-98,2006.

Monteiro, R. O. C.; Coelho, R. D.; Melo, P. C. T.; Ferraz, P.; Chaves, S. W. P. Aspectos produtivos e de qualidade do melão sob gotejo subterrâneo e mulching plástico. Revista Acta Science, v.29, p.453-457, 2007.

Ponciano, N. J.; Constantino, C. O. R.; Souza, P. M.; Detmann, E. Avaliação econômica da produção de abacaxi (Ananas comosus L.) cultivar Pérola na região Norte Fluminense. Revista Caatinga, v.19, p.82-91, 2006.

Reichardt, K. Processos de transferência no sistema solo-plantaatmosfera. Campinas : Fundação Cargill, 1985. 445p.

Rotondano, A. K. F.; Melo, B. Irrigação na cultura do abacaxizeiro.12 Out. 2011. <http://www.fruticultura.iciag. ufu.br/irriga7.html>.

Silva, C. A.; Silva, C. J. Irrigação na cultura do abacaxizeiro. Revista Científica Eletrônica de Agronomia, v.9, p.1-15, 2006.

Souza, C. B.; Silva, B. B. da; Azevedo, P. V. de. Crescimento e rendimento do abacaxizeiro nas condições climáticas dos Tabuleiros Costeiros do Estado da Paraíba. Revista Brasileira de Engenharia Agrícola e Ambiental, v.11, p.134141, 2007.

Souza, C. B.; Silva, B. B. da; Azevedo, P. V. de; Silva, V. de P. R. da. Fluxos de energia e desenvolvimento da cultura do abacaxizeiro. Revista Brasileira Engenharia Agrícola e Ambiental, v.12, p.400-407, 2008.

Souza, O. P.; Teodoro, R. E. F; Melo, M.; Torres, J. L. R. Qualidade do fruto e produtividade do abacaxizeiro em diferentes densidades de plantio e lâminas de irrigação. Pesquisa Agropecuária Brasileira, v.44, p.471-477, 2009.

Souza, O. P.; Zanini, J. R.; Torres, J. L. R.; Barreto, A. C.; Souza, E. L. C. S. Produção e qualidade física dos frutos do abacaxi sob diferentes lâminas e freqüências de irrigação. Revista Irriga, v.17, p.534-546, 2012. 